\title{
Importância das orientações em saúde para o desenvolvimento infantil e o aleitamento materno no primeiro ano de vida
}

\section{The importance of health guidelines for child development and breastfeeding in the first year of life}

\author{
Raphaela Barroso Guedes-Granzotti ${ }^{1}$, Kelly da Silva², Carla Patrícia Hernandez \\ Alves Ribeiro César ${ }^{3}$, Rodrigo Dornelas ${ }^{4}$, Larissa Santos Souza ${ }^{5}$, Larissa Santos \\ de Jesus $^{6}$, Taisa Ribeiro de Souza Oliveira ${ }^{7}$, Danielle Ramos Domenis ${ }^{8}$
}

http://dx.doi.org/10.11606/issn.2238-6149.v31i1-3p1-8

Guedes-Granzotti RB, Silva K, César CPHAR, Dornelas R, Souza LS, Jesus LS, Oliveira TRS, Domenis DR. Importância das orientações em saúde para o desenvolvimento infantil e o aleitamento materno no primeiro ano de vida. Rev Ter Ocup Univ São Paulo. 2020 jan.-dez.;31(1-3):1-8.

RESUMO: Objetivo: determinar o efeito das orientações em saúde para o desenvolvimento infantil e aleitamento materno no primeiro ano de vida. Método: estudo longitudinal ( $\mathrm{n}=45$ crianças), sendo 31 pertencentes ao grupo não exposto e 14 ao exposto, sendo este último acompanhado com visitas domiciliares mensais do nascimento ao $12^{\circ}$ mês de vida, recebendo informações sobre saúde materno-infantil. Foi utilizado o Teste de Triagem Denver II para acompanhamento do desenvolvimento neuropsicomotor e um questionário para avaliação das variáveis do estudo. Resultados: $64,3 \%$ do grupo exposto e $45,2 \%$ do não exposto foram amamentados até o primeiro ano de vida, sem diferenças significantes. Quanto à transição alimentar, a única relevância estatística foi a introdução de alimentos sólidos, ocorrendo em período adequado para o exposto e antecipado para o não exposto. Houve introdução precoce de líquidos e pastosos para ambos os grupos. Quanto ao desenvolvimento neuropsicomotor, houve maior número de sujeitos além do esperado para a idade e tendência de melhor coordenação motora fina no grupo exposto (sem diferenças estatísticas). Conclusão: as orientações evidenciaram que intervenções em saúde na primeira infầncia, mesmo para grupos que não são de risco, proporcionam oportunidades para um desenvolvimento pleno.

DESCRITORES: Educação em saúde; Desenvolvimento infantil; Aleitamento materno; Cuidado da criança.
Guedes-Granzotti RB, Silva K, César CPHAR, Dornelas R, Souza LS, Jesus LS, Oliveira TRS, Domenis DR. The importance of health guidelines for child development and breastfeeding in the first year of life. Rev Ter Ocup Univ São Paulo. 2020 Jan.-Dec.;31(1-3):1-8.

ABSTRACT: Objective: to determine the effect of health guidelines on child development and breastfeeding in the first year of life. Method: a longitudinal study ( $\mathrm{n}=45$ children), 31 belonging to the unexposed group, and 14 to the exposed group, the latter being accompanied with monthly home visits from birth to the 12th month of life, collecting information on maternal and child health. The Denver II Screening Test was used to monitor neuropsychomotor development and a questionnaire to assess the study variables. Results: $64.3 \%$ of the exposed group and $45.2 \%$ of the unexposed group were breastfed until the first year of life, with no significant differences. As for the food transition, the only statistical relevance was the introduction of solid foods, occurring in an appropriate period for the exposed group and anticipated for the unexposed group. There was an early introduction of liquids and pasty foods for both groups. As for neuropsychomotor development, there was a greater number of subjects than expected for age and a trend towards better fine motor coordination in the exposed group (without statistical differences). Conclusion: the guidelines showed that health interventions in early childhood, even for groups that are not at risk, provide opportunities for full development.

KEYWORDS: Health education; Child development; Breast feeding; Child care.

Agência de Fomento: CNPq (Universal processo 455135:2014-2)

1. Fonoaudióloga, Mestre e Doutora em Neurociências - USP. Professora Associada do Departamento de Fonoaudiologia da Universidade Federal de Sergipe. https://orcid.org/0000-0002-9064-439X. E-mail: raphaelabgg@gmail.com.

2. Fonoaudióloga, Mestre e Doutora em Ciências - USP. Professora Adjunta do Departamento de Fonoaudiologia da Universidade Federal de Sergipe. https://orcid.org/ 0000-0002-9193-7282. E-mail: kelly.fonoufs@gmail.com.

3. Fonoaudióloga, Mestre e Doutora em Distúrbios da Comunicação Humana - UNIFESP. Pós-doutora em Expressão Facial da Emoção pela Universidade Fernando Pessoa, Portugal. Professora Adjunta do Departamento de Fonoaudiologia da Universidade Federal de Sergipe. https://orcid.org/0000-0002-9439-9352. E-mail: carlacesar@globo.com.

4. Fonoaudiólogo, Mestre em Ciências da Saúde pela UNB, Doutor em Fonoaudiologia pela PUC-SP. Professor Adjunto do Departamento de Fonoaudiologia da Universidade Federal do Rio de Janeiro. https://orcid.org/0000-0002-9710-5751. E-mail: rdgdornelas@gmail.com.

5. Fonoaudióloga do Centro Especializado em Reabilitação de Alagoinhas, Bahia. https://orcid.org/0000-0002-6483-0949. E-mail: ss_larissa@outlook.com.

6. Fonoaudióloga residente no programa de Residência Multiprofissional em Saúde da Família da Universidade Federal de Sergipe. https://orcid.org/0000-0001-6301-7115. E-mail: la_larissaa@outlook.com.

7. Fonoaudióloga do Hospital Geral Roberto Santos; membro da Clínica Método de Desenvolvimento Humano. Especialista em Fonoaudiologia Hospitalar com ênfase em Neonatologia e Pós-graduada em Análise do Comportamento Aplicada ao Autismo. https://orcid.org/0000-0001-6358-3897. E-mail: ribeirotaisa16@gmail.

8. Fonoaudióloga, Mestre e Doutora em Ciências pela USP. Professora Adjunta do Departamento de Fonoaudiologia da Universidade Federal de Sergipe. https://orcid.org/00000002-9867-0066. E-mail: drdomenis@gmail.com.

Endereço para correspondência: Raphaela Barroso Guedes-Granzotti. Depto. Fonoaudiologia, Universidade Federal de Sergipe, Cidade Univ. Prof. José Aloísio de Campos. Av. Marechal Rondon, s/n, Jd. Rosa Elze, São Cristóvão, Sergipe. CEP: 49100-000. E-mail raphaelabgg@gmail.com. 


\section{INTRODUÇÃO}

alimentação e a nutrição concedida à criança
nos primeiros anos de vida repercutem ao longo de todo o seu crescimento ${ }^{1}$. O leite materno proporciona diversos benefícios ao bebê, pois garante o adequado suprimento nutricional, imunológico, desenvolvimento do sistema estomatognático, bem como experiências emocionais decorrentes da criação de vínculo entre a díade mãe-bebê , essenciais para o desenvolvimento da criança, como afeto, segurança e acolhimento ${ }^{2,3}$.

Estudos evidenciaram que o aleitamento materno (AM) interfere no desenvolvimento humano, da infância à vida adulta, sendo considerado o melhor alimento para o desenvolvimento encefálico. Os diferentes nutrientes e a sua composição geral contribuem para o amadurecimento cortical, por meio de ações químicas e funcionais, influenciando efetivamente no desenvolvimento neuropsicomotor infantil ${ }^{4}$. Sendo assim, o Ministério da Saúde do Brasil ${ }^{1}$ preconiza que o AM ocorra exclusivamente até o sexto mês e, posteriormente, de modo complementar, até dois anos de vida ou mais.

A partir dos seis meses está indicada a introdução da alimentação complementar estimulando sempre a manutenção do aleitamento materno. Todo o preparo da musculatura orofacial acontece durante a amamentação, pois ao sugar o leite no seio da mãe o lactente estimula de forma equilibrada todas as funções neuromusculares da região orofacial ${ }^{5}$ adequando assim a musculatura, de forma lenta e gradativa, para a necessidade do consumo de outras consistências. Sendo assim, a introdução alimentar tem o intuito de aumentar a densidade energética da dieta e seu subsídio nutricional, além de dar continuidade ao fortalecimento e crescimento das estruturas ósseas da face e o fortalecimento dos músculos do sistema estomatognático ${ }^{6}$.

Segundo o Ministério da Saúde ${ }^{1}$ é a partir dos seis meses, mantendo-se o aleitamento materno, que se pode dar início à introdução de novos alimentos, atendo-se para uma oferta com diversidade de cores, sabores, texturas e cheiros. É nessa idade que as crianças apresentam maturidade fisiológica e neurológica, como a atenuação do reflexo de protrusão da língua e a produção de enzimas digestivas em quantidades suficientes, habilitando-as a receber outros alimentos além do leite materno. Inicialmente os alimentos devem ser oferecidos na consistência pastosa, a partir dos nove meses em pequenos pedaços e, aos doze meses, na mesma consistência com que são consumidos pela família ${ }^{7}$.

Essa transição alimentar caracteriza um período de grande vulnerabilidade, no qual, além das modificações alimentares haverá também alteração do modo ou utensílio utilizado para a oferta da mesma. Para tanto, a introdução alimentar é uma prática que deve ser realizada de forma adequada, pois está diretamente relacionada com complexos aspectos biológicos, econômicos, culturais e sociais que podem repercutir no estado global da criança ${ }^{2}$.

A inserção precoce de outras substâncias, sem o adequado desenvolvimento do sistema motor oral e digestório, pode ocasionar infecções gastrointestinais, comprometimento do sistema imunológico e acarretar a desnutrição, pois o suprimento nutricional exigido pela criança não é totalmente ofertado ${ }^{6}$. Além disso, esta prática pode reduzir a duração do aleitamento materno e até dificultar a absorção de importantes nutrientes presentes no leite, podendo assim acarretar atraso no desenvolvimento dos aspectos neuropsicomotores infantis ${ }^{8,9}$, embora sobre essa associação (aleitamento materno e desenvolvimento neuropsicomotor) ainda não haja consenso na literatura ${ }^{10}$.

De forma semelhante, a introdução de novos alimentos também não deve ocorrer de forma tardia, pois acarreta desaceleração no crescimento da criança, já que só o leite materno não atende mais as necessidades energéticas; estando associado ao risco de desnutrição, devido à deficiência de energia, ferro, zinco, proteína e vitamina $\mathrm{A}$, além de uma maior dificuldade na aceitação alimentar futura, aumento dos riscos de desnutrição infantil e deficiência de micronutrientes ${ }^{4,14}$.

Desta forma, faz-se evidente a importância do incentivo ao aleitamento materno e a introdução alimentar de forma correta, tendo em vista que se realizados de maneira incorreta podem comprometer o desenvolvimento neuropsicomotor da criança. Frente ao exposto, o objetivo deste trabalho foi determinar o efeito de orientações em saúde infantil acerca do aleitamento materno, transição alimentar e desenvolvimento neuropsicomotor no primeiro ano de vida.

\section{MÉTODO}

Trata-se de um estudo longitudinal observacional tipo coorte, prospectivo e que faz parte de um projeto piloto denominado "Aleitamento materno e desenvolvimento infantil: práticas fonoaudiológicas para a promoção de saúde" que acompanhou o desenvolvimento de bebês em diversas áreas da saúde durante 12 meses. Aprovado pelo Comitê de Ética em Pesquisa (CAEE 42167415.8.0000.5546), seguiu as recomendações das Resoluções 466/2012 e 510/16 do Conselho Nacional de Saúde. Todos os responsáveis aceitaram livremente participar da pesquisa e foram informados de seus direitos como participante por meio do Termo de Consentimento Livre e Esclarecido, respeitando a decisão livre. 
A amostra composta por 45 participantes, de ambos os gêneros, divididos em dois grupos; sendo um composto por crianças que foram acompanhadas mensalmente por estudantes de fonoaudiologia durante o primeiro ano de vida e outro não, a saber:

- Grupo Estudo (GE): Formado por quatorze crianças nascidas na Maternidade Zacarias Junior e residentes na zona urbana do município de Lagarto/SE, recrutadas ao nascimento. Todas foram avaliadas nas primeiras 48 horas de vida - ainda na maternidade e receberam visitas domiciliares mensais de estudantes de fonoaudiologia para avaliação do desenvolvimento neuropsicomotor e a rotina alimentar até o décimo segundo mês de vida, constituindo 168 intervenções. A visita domiciliar foi a opção desta pesquisa tendo em vista que a literatura aponta para este tipo de intervenção como estratégia protetora da amamentação, principalmente na primeira semana de $v \operatorname{vida}^{15}$. Em todas as visitas as mães foram orientadas quanto a importância da amamentação, sobre a introdução adequada de alimentação complementar e em como estimular o desenvolvimento infantil. Este grupo participou do seguimento da coorte;

- Grupo não exposto (GNE): Formado por 31 crianças nascidas no ano de 2016 também na Maternidade Zacarias Junior e residentes na zona urbana do município de Lagarto/SE. As crianças foram selecionadas a partir do banco de dados da maternidade pareados com o grupo GE, com relação ao tipo de parto e ao sexo. Todas as crianças foram avaliadas em uma única visita domiciliar quanto ao desenvolvimento neuropsicomotor e a rotina alimentar no décimo segundo mês de vida, não participando das visitas mensais, denominado assim por "não exposto".

Como forma de evitar o efeito de variáveis de confusão, foram consideradas aquelas que podem interferir no desenvolvimento infantil, em ambos os grupos sendo os fatores de inclusão: crianças com peso ao nascimento acima de $2500 \mathrm{~g}$, tempo de gestação superior a 37 semanas, mães maiores de dezoito anos e nascimentos a termo (entre $37 \mathrm{e}$ 42 semanas de idade gestacional). Foram excluídas aquelas que apresentaram infecções e/ou complicações perinatais, doenças neurológicas, doenças metabólicas, desnutrição proteico calórica, malformações, intercorrências durante o parto e no período neonatal, internação prolongada, crianças institucionalizadas ou que por algum motivo não moravam com suas mães, bem como aquelas que realizaram qualquer tipo de acompanhamento fonoaudiológico. Para tanto, essas variáveis foram consultadas no momento das entrevistas com as mães do grupo estudo e, para o grupo não exposto, pela verificação dos prontuários da maternidade.

Para avaliar o risco para atraso no desenvolvimento neuropsicomotor foi realizado, em todas as visitas, o Teste de Triagem de Desenvolvimento de Denver II ${ }^{12}$. Este teste contém 125 itens que analisam as áreas da Linguagem (verifica aspectos expressivos e de compreensão e o uso da linguagem), Pessoal-Social (avalia a sociabilidade da criança), Motor Grosseiro (observa e analisa movimentos motores realizados pela musculatura ampla), e Motor Fino Adaptativo (verifica se a criança consegue manipular objetos pequenos e se apresenta coordenação viso-manual). Cada item do instrumento é representado por uma barra que mostra a idade em que as crianças (nos seguintes percentuais: $25 \%$, $50 \%, 75 \%$ e $90 \%$ ) podem realizar as áreas testadas e atribui-se um escore (passou, falhou, recusou ou sem oportunidade) por item. Posteriormente o resultado de cada área foi categorizado como normal, de risco, avançado e não testável seguindo as orientações do teste ${ }^{16}$. Foram considerados alguns fatores de exclusão temporária que poderiam afetar o desempenho da criança no rastreio do desenvolvimento neuropsicomotor, como sono, fadiga, adoecimento, febre ou medo. Durante a triagem, o teste foi interrompido caso a criança demonstrasse cansaço sendo retomado em outro momento sem prejuízo para a sua validade.

Também foi utilizado no estudo um questionário ${ }^{13}$ que possuía dezoito questões que abordaram dados relacionados ao perfil pessoal, aspectos sociodemográfico, de saúde pregressa e puericultura, com foco em dados sobre o AM, hábitos orais e introdução alimentar. Alguns itens foram preenchidos em uma única visita e os referentes à saúde e alimentação foram preenchidos de acordo com o seguimento das visitas domiciliares. A classificação do tipo de AM adotada neste estudo seguiu as definições e recomendações da Organização Mundial da Saúde ${ }^{14}$.

As intervenções fonoaudiológicas iniciaram-se na maternidade e, com o grupo estudo, foram mensais, em dia e horário previamente agendados com as responsáveis pelos sujeitos do grupo estudo, contando com atividades dialogadas sobre a importância do aleitamento materno para o fortalecimento do vínculo mãe-bebê, para o desenvolvimento da musculatura orofacial e global da criança (incluindo o cognitivo, neuropsicomotor, emocional, linguagem, entre outros), sobre os seus nutrientes, a transição alimentar, sobre as alterações estruturais e funcionais do sistema estomatognático, entre outros. Durante as atividades, materiais educativos foram utilizados para facilitar a compreensão dos temas abordados. A amamentação também foi observada sempre que possível. 
A atividade era finalizada com respostas às dúvidas e se havia algum tema de preferência para o próximo encontro, a fim de personalizar tais encontros, que tinham a duração aproximada de uma hora. Todas as atividades realizadas foram respaldadas pela literatura da área.

Os resultados foram preenchidos em planilha do software Excel (pacote Microsoft ${ }^{\circledR}$ Office). Para análise descritiva foram utilizadas as frequências absolutas, frequência relativas e medidas de tendência central e de dispersão. Para análise inferencial foram utilizados testes de associação comparando os resultados do questionário entre o grupo não exposto e experimental. Foram utilizados os testes Qui-quadrado e Mann-Whitney (para comparação de dados ordinais) e estipulado o nível de significância de $5 \%(p<0,05)$.

\section{RESULTADOS}

A Tabela 1 apresenta o perfil dos dois grupos quanto ao sexo das crianças, a idade e escolaridade das mães e se trabalham fora de casa, o tipo de parto e a renda familiar da família.

Tabela 1 - Caracterização das participantes

\begin{tabular}{llcc}
\hline & & Grupo Estudo & Grupo Não Exposto \\
\hline \multirow{2}{*}{ Sexo das crianças } & Masculino & $8(57,2 \%)$ & $15(48,4 \%)$ \\
& Feminino & $6(42,8 \%)$ & $16(51,6 \%)$ \\
\hline \multirow{2}{*}{ Idade das mães } & & $18-38$ anos & $18-42$ anos \\
& & $\mathrm{M}=27,3( \pm 5,2)$ & $\mathrm{M}=27,8( \pm 6,7)$ \\
\hline \multirow{2}{*}{ Tipo de parto } & Vaginal & $9(64,3 \%)$ & $17(54,8 \%)$ \\
\hline \multirow{2}{*}{ Escolaridade das mães } & Cesariana & $5(35,7 \%)$ & $14(45,2 \%)$ \\
& Ens. Fundamental Incomp. & $13(41,9 \%)$ \\
\hline Renda Familiar & Ens. Médio Completo & $5(37,7 \%)$ & $10(32,3 \%)$ \\
Trabalho fora de casa & Ens. Superior Completo & $5(37,7 \%)$ & $8(25,8 \%)$ \\
\hline
\end{tabular}

Análise Descritiva

Legendas: M-Média; Ens. Fund. Incomp - Ensino Fundamental Incompleto; Ens. Médio Completo - Ensino Médio Incompleto; Ens. Superior Completo Ensino Superior Completo.

A Tabela 2 apresenta a frequência absoluta e relativa de crianças em aleitamento materno aos seis meses e 12 meses de idade, nos grupos com acompanhamento e sem acompanhamento.

É possível observar na Tabela 2 que no grupo com acompanhamento todas as crianças que estavam amamentando aos seis meses continuaram sendo amamentadas até os dozes meses de vida. Em contrapartida, no grupo sem acompanhamento entre o sexto e o décimo segundo mês ocorreu uma redução de $25 \%$ no número de crianças em aleitamento materno. Apesar da referida diferença, não houve relevância estatística entre os valores obtidos nos grupos investigados.

Quanto à introdução alimentar a Tabela 3 traz os valores absolutos e relativos de crianças com introdução adequada e inadequada para a idade. Ao se comparar os grupos com e sem acompanhamento observou-se diferença significativa quanto a introdução do sólido, para as demais consistências não houve diferenças entre os grupos

A Tabela 4 apresenta os resultados do desenvolvimento neuropsicomotor infantil entre os grupos com e sem acompanhamento.

Tabela 2 - Comparação entre o Aleitamento Materno, no $6^{\circ}$ e $12^{\circ}$ mês, entre os grupos estudados.

\begin{tabular}{lccccc}
\hline & \multicolumn{2}{c}{ Grupo Estudo } & \multicolumn{2}{c}{ Grupo Não Exposto } & \multirow{2}{*}{ P valor } \\
\cline { 2 - 5 } & $\mathbf{6}^{\mathbf{0}}$ Mês & $\mathbf{1 2}^{\mathbf{0}}$ Mês & $\mathbf{6}^{\mathbf{0}}$ Mês & $\mathbf{1 2}^{\mathbf{0}}$ Mês & \\
\hline Manutenção do aleitamento & $9(64,3 \%)$ & $9(64,3 \%)$ & $20(64,5 \%)$ & $15(45,4 \%)$ & $\mathrm{p}>0,05$ \\
Interrupção do aleitamento & $5(35,7 \%)$ & $5(35,7 \%)$ & $11(35,5 \%)$ & $16(51,6 \%)$ & $\mathrm{p}>0,05$ \\
\hline
\end{tabular}

Teste Qui-quadrado 
Guedes-Granzotti RB, et al. Importância das orientações em saúde para o desenvolvimento infantil . Rev Ter Ocup Univ São Paulo. 2020 jan.-dez:;31(1-3):1-8.

Tabela 3 - Comparação entre a introdução alimentar complementar entre os grupos estudados.

\begin{tabular}{lccccc}
\hline & \multicolumn{2}{c}{ Grupo Exposto } & \multicolumn{2}{c}{ Grupo Não Exposto } & \multirow{2}{*}{ P valor } \\
\cline { 2 - 5 } & Adequado & Inadequado & Adequado & Inadequado & \\
\hline Introdução de Líquido & $3(21,4 \%)$ & $11(78,6 \%)$ & $6(19,4 \%)$ & $25(80,6 \%)$ & $\mathrm{p}>0,05$ \\
Introdução de Pastoso & $8(57,1 \%)$ & $6(42,9 \%)$ & $12(38,7 \%)$ & $19(61,3 \%)$ & $\mathrm{p}>0,05$ \\
Introdução de Sólido & $14(100 \%)$ & $0(0 \%)$ & $13(41,9 \%)$ & $18(58,1 \%)$ & $\mathrm{p}<0,001 *$ \\
\hline
\end{tabular}

Teste Qui-quadrado

Tabela 4 - Comparação do desenvolvimento neuropsicomotor entre os grupos estudados.

\begin{tabular}{|c|c|c|c|c|c|c|c|}
\hline & \multicolumn{3}{|c|}{ Grupo Exposto } & \multicolumn{3}{|c|}{ Grupo Não Exposto } & \multirow{2}{*}{ P valor } \\
\hline & Avançado & Normal & Risco & Avançado & Normal & Risco & \\
\hline Pessoal Social & $4(28,6 \%)$ & $9(64,3 \%)$ & $1(7,1 \%)$ & $2(6,4 \%)$ & $26(83,9 \%)$ & $3(9,7 \%)$ & 0,2 \\
\hline Linguagem & $3(21,4 \%)$ & $11(78,6 \%)$ & $0(0 \%)$ & $2(6,4 \%)$ & $26(83,9 \%)$ & $3(9,7 \%)$ & 0,8 \\
\hline Motor grosseiro $^{1}$ & $4(28,6 \%)$ & $10(64,3 \%)$ & $0(0 \%)$ & $2(6,4 \%)$ & $26(83,9 \%)$ & $3(9,7 \%)$ & 0,5 \\
\hline Motor fino & $7(50 \%)$ & $7(50 \%)$ & $0(0 \%)$ & $3(9,7 \%)$ & $25(80,6 \%)$ & $3(9,7 \%)$ & 0,08 \\
\hline
\end{tabular}

Teste Mann-Whitney

Legenda: ${ }^{1}$ uma criança do grupo com acompanhamento não foi possível testar o motor grosseiro.

\section{DISCUSSÃO}

Nos primeiros anos de vida o desenvolvimento e crescimento infantil dependem de diversos aspectos, tanto ambientais quanto biológicos, sendo essencial que durante os primeiros seis meses de vida a criança seja alimentada única e exclusivamente pelo leite materno ${ }^{1}$, tendo em vista que tal leite apresenta as proporções adequadas de nutrientes para o lactente, melhor digestibilidade, nutrição de alta qualidade entre outros benefícios ${ }^{19}$. Todavia, o ato de amamentar pode ser influenciado por inúmeros fatores intrínsecos e extrínsecos, resultando no desmame precoce ${ }^{1}$.

Neste estudo o desmame precoce ocorreu nas mesmas proporções em ambos os grupos, sendo necessário analisar os aspectos que podem ter influenciado esses achados. Com relação à influência da idade materna no desmame precoce a literatura é controversa. Em uma pesquisa foi apontada a idade maior que 20 anos como um fator protetor do $\mathrm{AM}^{15}$, enquanto outra pesquisa não identificou associação entre a idade e o desmame precoce ${ }^{16}$. Contudo, como um dos critérios de inclusão utilizados era a genitora ser maior de idade, não haveria possibilidade de este aspecto influenciar na ocorrência de desmame precoce encontrado no estudo.

O nível de escolaridade da progenitora é outra variável que é bastante discutida na literatura ${ }^{15,16}$. Nesta pesquisa o índice de mães sem instrução ou com o ensino fundamental incompleto eram expressivos em ambos os grupos estudados; demonstrando homogeneidade que podem justificar os resultados obtidos. A grande maioria das pesquisas relacionam o menor nível de escolaridade com a menor duração do aleitamento exclusivo ${ }^{16}$; entretanto, outros estudos não encontraram relação entre as atividades ${ }^{17}$.

Quanto à renda per capita das famílias, em ambos os grupos, foi inferior a um salário mínimo. Estudos indicam a relação entre desmame precoce e baixa renda ${ }^{18}$, aspecto este que acarretaria menor acesso ao pré-natal adequando e consequentemente; limites ao acesso às informações e ao acompanhamento não só durante a gestação, mas também após; aumentando a introdução de alimentos complementares incorretos e do desmame precoce.

O tipo de parto também é visto como outro fator de risco para a interrupção do aleitamento antes do sexto mês. Essa colocação é justificada, pois na cesariana a mulher passa por diversos procedimentos que afetaria na prática do aleitamento materno; como a aplicação de anestesia, uso de medicamentos e falta de preparo do corpo para a produção do leite; situações estas que não são identificadas em casos de partos normais. Além disso, o vínculo afetivo estabelecido entre a mãe e bebê facilita o estabelecimento do processo de lactação ${ }^{19}$. Nesta pesquisa a porcentagem de partos cesáreos foi expressiva em ambos os grupos, sendo um pouco maior no GE.

Foi possível verificar homogeneidade entre os grupos quanto aos aspectos socioeconômicos o que pode explicar a 
ausência de resultados significativos, quando comparamos estas variáveis entre eles. Demonstrando também que os achados indicam que os dois grupos apresentaram importantes fatores de risco para o desmame precoce.

Certamente os primeiros meses de vida do bebê são os mais difíceis tanto para o bebê que está se adaptando ao mundo, quanto para a mãe (principalmente se for primípara) e família, devidos aos cuidados que devem ser intensificados e destinados para a sobrevivência do novo ser. Oliveira et al. ${ }^{13}$ evidenciaram que os lactentes que permaneceram em aleitamento materno até o sexto mês de vida apresentaram melhor desenvolvimento neuropsicomotor e menor incidência de hábitos orais deletérios, não havendo diferença quanto à introdução alimentar.

Em relação à introdução de novos alimentos, o bebê que tem alimentos introduzidos de forma precoce à sua dieta, procura saciar todas as suas necessidades psicológicas e exercitar a musculatura orofacial através da sucção não nutritiva. Estes hábitos prejudicam o desenvolvimento das estruturas e funcionalidade do sistema estomatognático ${ }^{20}$, a depender da intensidade, frequência e duração. Além do exposto, os hábitos orais deletérios têm a tendência a se instalar principalmente em bebês que não receberam o aleitamento materno ${ }^{5}$.

Após o sexto mês recomenda-se que outras substâncias comecem a ser inseridas na dieta do bebê, com variações quanto à frequência, quantidade e consistência; líquida, pastosa e sólida. Essa inserção é bastante complexa e deve ocorrer de modo gradual, pois sofre influência de diversos fatores como o contexto familiar, econômico e sociocultural. O consumo precoce de água, chás e sucos são mais comuns, informação compatível com os achados da pesquisa na qual a maioria introduziu líquidos antes do sexto mês de $v^{\text {vida }}{ }^{2}$. Em relação à introdução do alimento sólido, poucos estudos investigam esse tema. Doub et al. ${ }^{21}$ constataram que o momento da introdução de alimentos sólidos é uma escolha materna e esteve correlacionado positivamente com a idade das mães, grau de instrução, amamentação aos quatro meses, resposta autorreferida às pistas de fome e saciedade dos bebês e correlacionada negativamente com o índice de massa corporal pré-gestacional das mães, às crenças sobre os alimentos sólidos antes dos seis meses de idade e à reatividade motora temperamental dos bebês. Podemos inferir, pelos resultados obtidos e diferenças entre os grupos, que as orientações fonoaudiológicas fornecidas às mães do grupo estudo, no período de transição alimentar, fomentaram um maior conhecimento materno acerca dos comportamentos infantis, principalmente aqueles associados à fome, bem como sore suas crenças, de forma a evitar a introdução precoce dessa consistência alimentar, ratificando o exposto pela literatura.

Outro aspecto a ser salientado, é que por influência de suas mães, as progenitoras acabam acreditando que o leite é fraco ou insuficiente para saciar a fome e a sede da criança. Conforme observado por Oliveira et al. ${ }^{22}$ a avó materna se configura como um fator importante para o desmame precoce e a manutenção e a manutenção do aleitamento materno, principalmente o exclusivo, pois transmitem um conhecimento empírico, de seus antepassados, baseada no senso comum.

Nesses casos o nosso trabalho focou em promover o fortalecimento do vínculo e incentivar a manutenção do aleitamento materno misto. Além disso, orientações foram dadas quanto à introdução correta das consistências. Com isso, as substâncias pastosas foram inseridas a dieta do bebê de forma adequada, na maioria dos casos; já a sólida, ocorreu corretamente em todos que compunham o grupo estudo e a maioria dos integrantes do grupo não exposto.

No decorrer do primeiro ano de vida um dos fatores primordiais para a promoção do desenvolvimento cerebral apropriado é a forma com que essa criança foi nutrida. Assim, a alimentação complementar merece atenção especial nesta faixa etária. Caso seja realizada da maneira inadequada pode acarretar desnutrição e deficiência de nutrientes que geram danos ao sistema nervoso central e, consequentemente, atraso no desenvolvimento neuropsicomotor infantil ${ }^{8}$, aspecto que foi investigado por meio da aplicação mensal do Denver II no grupo estudo.

A aprendizagem e aquisição de habilidades cognitivas, socioemocionais, sensoriais, linguagem, motora ampla e fina são influenciadas por diversos fatores e não ocorrem da mesma maneira. A exposição e incidência da estimulação nos primeiros anos de vida podem alterar, limitando ou facilitando, o desenvolvimento neuropsicomotor infantil ${ }^{23}$.

O melhor desenvolvimento do GE pode estar relacionado à oportunidade do brincar, pois sabendo da relevância desta prática, em todas as visitas realizadas os pesquisadores frisaram a importância da estimulação do desenvolvimento infantil, sendo os jogos e brincadeiras em geral as atividades que contribuem para o pleno desenvolvimento infanti $1^{24}$. Sugestões foram dadas para que por meio do brincar as habilidades motoras, linguísticas e pessoal social estivessem sendo trabalhadas. Tais recomendações mostraram-se relevantes já que nenhuma criança apresentou risco nos aspectos avaliados.

A atuação fonoaudiológica faz-se necessária de forma conjunta com a equipe interdisciplinar para tentar alcançar resultados mais positivos em relação aleitamento 
materno exclusivo. Uma vez que, segundo o DATASUS, a cada mil nascidos vivos, $57,8 \%$ realizaram aleitamento exclusivo no município de Lagarto ${ }^{25}$. Diante disso, sendo o fonoaudiólogo profissional capacitado para atuar em tal área, é imprescindível a sua participação em práticas de incentivo ao aleitamento exclusivo e na prevenção dos distúrbios da comunicação, assim como na promoção do desenvolvimento infantil, no acompanhamento das mães que realizaram o desmame precoce em seus filhos ou que estão na etapa de introdução alimentar complementar.

Observou-se que o acompanhamento fonoaudiológico proporcionou efeitos benéficos aos participantes (mães, bebês, discentes e docentes envolvidos). Estes resultados ratificam as diretrizes mundiais que afirmam que o acesso à informação garante o sucesso na amamentação.

\section{CONCLUSÃO}

Por meio desta pesquisa observou-se a importância do trabalho com foco no incentivo ao aleitamento exclusivo, à transição alimentar e ao desenvolvimento neuropsicomotor. De forma geral, houve melhoras nos aspectos trabalhados no grupo que recebeu o acompanhamento fonoaudiológico após a ação de educação materna para o desenvolvimento infantil, tendo em vista que tais intervenções propiciaram a construção saudável da maternidade, a escuta ativa das dificuldades do desempenho desse papel, reflexão acerca da influência cultural nos cuidados da primeira infância e a possibilidade de desenvolvimento infantil pleno. Ademais, intervenções em saúde para bebês, mesmo que não sejam de risco, proporcionam a todos os envolvidos (mãe e bebê) oportunidades para o incentivo à saúde no primeiro ano de vida.

Contribuição dos autores: $R B G G$ e $D R D$ - Concepção do projeto, análise e interpretação dos dados, redação e revisão do texto. $K S$, $R D e$ CPHARC - Análise e interpretação dos dados, redação e revisão do texto. LSS, LSJ e TRSO - Coleta de dados, análise e interpretação dos dados, redação e revisão do texto. Todos os autores aprovaram a versão final.

\section{REFERÊNCIAS}

1. Brasil. Ministério da Saúde. Secretaria de Atenção Primária à Saúde. Departamento de Proteção da Saúde. Guia alimentar para crianças menores de dois anos de idade. Brasília; 2019.

2 Amaral LJX, Sales SS, Cavalho DPSRP, Cruz GKP, Azevedo IC, Ferreira Júnior MA. Fatores que influenciam na interrupção do aleitamento materno exclusivo em nutrizes. Rev Gaúcha Enferm. 2015;36:127-34. https://doi.org/10.1590/ 1983-1447.2015.esp.56676.

3. Paixão MCDS, Goés ACF, Del Raso A, Leal MAF. A amamentação sob o olhar das puérperas e as influências do meio sociofamiliar no processo de vinculação mãe-bebê. Contextos Clín. 2019;12(3): 863-80. http://dx.doi.org/10.4013/ctc.2019.123.08.

4. Clark KM, Castilho M, Calatroni A, Walter T, Cayazzo M, Pino P, Lozoff B. Breastfeeding and mental and motor development at 5 1/2 years. Ambul Pediatr. 2006;6(2):65-71. https://doi.org/10.1016/j.ambp.2005.11.003.

5. Souza SNDH, Migoto MT, Rossetto EG, Mello DF. Prevalência de aleitamento materno e fatores associados no município de Londrina - PR. Acta Paul Enferm. 2012;25(1):29-35. http://dx.doi.org/10.1590/S0103-21002012000100006.

6. Ximenes LB, Moura JG, Oriá MOB, Martins MC, Almeida PC, Carneiro EP. Práticas alimentares e sua relação com as intercorrências clínicas de crianças de zero a seis meses. Esc Anna Nery Rev Enferm. 2010;14(2):377-85. http://dx.doi.org/ 10.1590/S1414-81452010000200023.
7. Weffort VRS, Escrivão MAMS, Oliveira FLC, Rocha HF, Mello ED, Mattos ÂP et al. Manual de orientação para a alimentação do lactente, do pré-escolar, do escolar, do adolescente e na escola. (acesso em 15 abr. 2020). Disponível em: http://www.sbp.com.br/ pdfs/14617a-PDManualNutrologia-Alimentacao.pdf.

8. Schincaglia RM, Oliveira AC, Sousa LM, Martins KA. Práticas alimentares e fatores associados à introdução precoce da alimentação complementar entre crianças menores de seis meses na região noroeste de Goiânia. Epidemiol Serv Saúde. 2015;24(3):465-74. http://dx.doi.org/10.5123/S167949742015000300012

9. Chiu W-C, Liao H-F, Chang P-J, Chen P-C, Chen YC. Duration of breastfeeding and risk of developmental delay in Taiwanese children: a nationwide birth cohort study. Paediatr Perinat Epidemiol. 2011;25:519-27. http://dx.doi.org/10.1111/j.13653016.2011.01236.x.

10. Michels KA, Ghassabian A, Mumford SL, Sundaram R, Bell EM, Bello SC, Yeung EH. Breastfeeding and motor development in term and preterm infants in a longitudinal US cohort. Am J Clin Nutr. 2017;106:1456-62. http://dx.doi.org/ 10.3945/ajen.116.144279.

11. Silva VAAL, Caminha MFC, Silva SL, Serva VMSBD, Azevedo PTACC, Batista Filho M. Aleitamento materno: indicadores e fatores associados à amamentação exclusiva num aglomerado urbano subnormal assistido pela Estratégia de Saúde da Família. J Pediatr. 2019;95(3):298-305. http://dx.doi.org/10.1016/j. jped.2018.01.004. 
12. Frankenburg FK, Dodds JB, Archer P, Bresnick B, Maschka P, Edelman N, Shapiro H. Denver II: Technical Manual and Training Manual. Denver: Denver Developmental Materials Inc.; 1990.

13. Oliveira TRS, Souza LS, Dornelas R, Domenis DR, Silva K, Guedes-Granzotti RB. Associação entre o aleitamento materno, introdução alimentar e desenvolvimento neuropsicomotor nos primeiros seis meses de vida. Distúrb Comum. 2017;29(2):262-73. https://doi.org/10.23925/2176-2724.2017v29i2p262-273.

14. World Health Organization (WHO). Indicators for assessing infant and young child feeding practices. Conclusions of consensus meeting held 6-8. Washington; 2007.

15. Margotti E, Epifanio M. Aleitamento materno exclusivo e a escala de autoeficácia na amamentação. Rev Rene. 2014;15(5):771-9. https://doi.org/10.15253/2175-6783.2014000500006

16. Apostolakis-Kyrus K, Valentine C, DeFranco E. Factors associated with breastfeeding initiation in adolescent mothers. J Pediatr. 2013;163(5):1489- 94. https://doi.org/10.1016/ j.jpeds.2013.06.027.

17. Caldeira AP, Goulart EM. A situação do aleitamento materno em Montes Claros, Minas Gerais: estudo de uma amostra representativa. J Pediatr. 2000;76: 65-72.

18. Sales-Costa R, Barroso GS, Cabral M, Castro MBT. Parental dietary patterns and social determinants of children's dietary patterns. Rev Nutr. 2016;29(4):483-93. https://doi.org/ $10.1590 / 1678-98652016000400004$.
19. Pereira CRVR, Fonseca VM, Oliveira MIC, Souza IEO, Mello RR. Avaliação de fatores que interferem na amamentação na primeira hora de vida. Rev Bras Epidemiol. 2013;16(2):525-34. https://doi.org/10.1590/S1415-790X2013000200026.

20. Araújo HRV, Carvalho MT, Imparato JCP, Pinchemel ENB. A importância do aleitamento materno no controle do desenvolvimento de hábitos deletérios: revisão de Literatura. Rev Multidiscip Psicol. 2019,13(47):1135-44. https://doi.org/ 10.14295/idonline.v13i47.2109

21. Doub AE, Moding KJ, Stifter CA. Infant and maternal predictors of early life feeding decisions. The timing of solid food introduction. Appetite. 2015;92:261-8. https://doi.org/10.1016/ j.appet.2015.05.028.

22. Oliveira CS, Locca FA, Carrijo MLZ, Garcia RATM. Amamentação e as intercorrências que contribuem para o desmame precoce. Rev Gaúcha Enferm. 2015;36:16-23. https://doi.org/10.1590/1983-1447.2015.esp.56766.

23. Costa EF, Cavalcante LIC, Dell'Aglio D. Language development profile of children in Belem, according to Denver Developmental Screening Test. Rev CEFAC. 2015;17(4):1090-102. https://doi.org/10.1590/1982-0216201517418514

24. Pena ALF. A psicomotricidade no desenvolvimento físico, cognitivo e social da criança. Rev Form Prat Docente. 2020;(3):54-64.

25. Brasil. Ministério da Saúde. Datasus: cadernos de informações de saúde Sergipe [cited 2019 Jun. 28], 2009. Available from: http://tabnet.datasus.gov.br/tabdata/cadernos/se.htm. 